because latencies may vary between subject groups that consistent nomenclature is essential. Evoked potentials are conventionally identified according to the polarity and modal latency of their peaks. Thus when potentials from patients and normal subjects are compared, like is compared with like, and changes in the characteristics of the potentials may be related to changes in the processes or structures generating them. Unfortunately, when the present results are quoted by future authors, confusion will arise. For instance, it would seem reasonable to suppose that in a paper on early cortical-evoked potentials, the wave labelled N1 (their Fig. 1) would refer to the initial negative-going cortical potential (which is conventionally called $\mathrm{N} 19$ or $\mathrm{N} 20$ since its modal latency is about $20 \mathrm{~ms}$ ). A peak corresponding to $\mathrm{N} 20$ is clearly present in Fig. 1 but is unlabelled and distinct from the peak labelled N1.

The authors note considerable variability both within and between subjects in the amplitudes of their cortical potentials. At least some of this variability is likely to arise from the type of stimulus used. Vibrotactile stimulation of the skin of the finger may excite impulses in a wide range of sensory fibres of differing conduction velocities (Iggo, 1982); together with the $10 \mathrm{~ms}$ duration of the stimulus this produces a diffuse and temporally dispersed afferent volley. Furthermore, spread of vibration is a potent stimulus to muscle receptors of the intrinsic muscles of the hand and forearm (Davies, 1987). Since the amplitude of excursion of the vibrator is not quoted in their paper, the extent of these effects cannot be assessed.

Finally, a further source of variability might arise from combining data from patients suffering different forms of schizophrenic illness. Until proved otherwise, it should not be assumed that sensory processing or its dysfunction is similar in acute $v$. chronic patients, or in those with positive v. negative symptoms.

COOPER, J. E., ANDrews, H. \& BArber, C. (1985) Stable abnormalities in the lateralisation of early cortical somatosensory evoked potentials in schizophrenic patients. British Journal of Psychiatry, 146, 585-593.

DAviEs, T. W. (1987) Contribution from hand muscle afferents to forearm muscle stretch reflexes in man. Journal of Physiology, 390, 258.

IGGo, A. (1982) Cutaneous sensory mechanisms. In The Senses (eds H. B. Barlow \& J. D. Mollon), pp. 369-408. Cambridge: Cambridge University Press.

Tress, K. H., Caudrey, D. J. \& Merta, B. (1983) Tactile-evoked potentials in schizophrenia. Interhemispheric transfer and drug effects. British Journal of Psychiatry, 143, 156-164.

Bexley Hospital

TEIFION DAviES

Old Bexley Lane

Bexley, Kent DA5 2BW

\section{Clozapine in patients with NMS}

SIR: Szabadi \& Cashman (Journal, April 1991, 158, 577) suggest that clozapine is the drug to consider when a patient has suffered neuroleptic malignant syndrome (NMS). However, it would be inappropriate to generalise their experience with clozapine to other patients who have suffered NMS. The first case report linking clozapine (in combination with lithium) to NMS appeared more than four years ago (Pope et al, 1986). This was followed by another case in which NMS was presumed to have resulted from a combination of clozapine and carbamazepine (Muller et al, 1988). There might have been some scepticism about these reports as clozapine by itself could not be implicated. More recently, a series of case reports suggested that clozapine alone can cause NMS (e.g. DasGupta \& Young, 1991). It would not be unfair to say that the strategy suggested by Szabadi \& Cashman (1991) has also been adopted in the past without recurrence of NMS. Despite its different structure and pharmacological profile, clozapine does affect the dopaminergic system; recently the gene for a human dopamine D4 receptor with high affinity for clozapine has been cloned (Van Tol et al, 1991). In view of these observations it may be rather premature to generalise about the potential safety of clozapine in patients with NMS who need further antipsychotic treatment.

DAsGuPTA, K. \& Young, A. (1991) Clozapine-induced neuroleptic malignant syndrome. Journal of Clinical Psychiatry, 52, 105-107. MULlER, T., BECKER, T. \& FritzE, J. (1988) Neuroleptic malignant syndrome plus carbamazepine. Lancet, 31, 1499-1500.

POPE, H. G., COLE, J. O., ChORAs, P. T., et al (1986) Apparent neuroleptic malignant syndrome with clozapine and lithium. Journal of Nervous and Mental Diseases, 174, 493-495.

Van Tol, H. H. M., Bunzow, J. R., Guan, H. C. el al (1991) Cloning of the gene for a human dopamine D4 receptor with high affinity for the antipsychotic clozapine. Nature, 350, 610-614.

The Maudsley Hospital

AdityanjeE

Denmark Hill

London SES 8 AZ

\section{Outcome indicators in mental handicap}

SIR: We read with interest Jenkins' article (Journal, October 1990,157,500-514), particularly on outcome indicators for mental handicap. Although we fully agree there is an urgent need for specific outcome indicators for mental health care, we were dismayed to see that many of the suggested indicators for mental handicap bore little relationship to the clinical practice of the 'psychiatry of mental handicap'. Many indicators given seemed to be more related to the clinical practices of obstetrics, paediatrics, 
community medicine, etc., aimed at the prevention and aetiology of mental handicap, rather than the practice of mental health care.

The author rightly states that people with a mental handicap are more prone to developing psychiatric disorders, and probably appreciates that there are vulnerability factors applicable to this group of people, such as social, emotional, environmental and physical causes. Thus some people with a mental handicap have additional special mental health needs and require specialist and generic mental health care services. The outcome indicators chosen should reflect an understanding and acknowledgement of these needs. The increased adoption and use of multiaxial classification systems (DSM-III-R, and the recently proposed multi-aspect assessment (Annes et al, 1991)) show the appreciation of the multiplicity of factors contributing to the development of psychiatric illness, behaviour disorders, personality disorder, and offending in people with a mental handicap. Similar approaches need to be extended to outcome measurements.

In reducing the incidence of mental handicap, we should also be aiming to reduce the incidence and prevalence of additional special mental health needs as well as reducing the related effects of adverse social and environmental factors. Therefore we need to relate outcomes to input and process activities. The latter indicators should aim at ensuring good quality comprehensive service provisions, including regular individualised client reviews with forward coordinated planning for both providers and clients across all age groups. More specifically, indicators of specialist mental health care input must include the availability of appropriate high quality community and hospital-based specialist psychiatric services for people with a mental handicap who need them. These should consist of assessment, day-patient and in-patient treatment, rehabilitation, and follow-up components.

Measurable outcomes of input and process activities should be ideally related to reduction of disability as well as enhancement of skills. Outcomes of mental health care input should take into account relapse and readmission rates. All indicators should be applicable to people with a mental handicap living in both the community and hospitals.

Further research, together with the practices of clinical audit, quality assurance, and consumer satisfaction initiatives, should contribute to the development of specific indicators relevant to clinical practice of mental handicap and mental health. A closer dialogue and collaboration between researchers, policy makers and clinicians is undoubtedly needed.
Anness, V., Bhat, A., Bouras, N., et al (1991) A multi-aspect assessment for people with a mental handicap. Psychiatric Bulletin, 15, 146.

\section{S. Gravestock}

G. HOLT

N. BOURAS

Section of Psychiatry of Mental Handicap

Guy's Hospital

London SE1 9RT

\section{Census-derived measures and planning}

SIR: Thornicroft's exploration of the relationship between treated mental disorder and census-derived measures of social deprivation clearly has important implications for the planning of mental health services (Journal, April 1991, 158, 475-484). The great advantage of census-derived indices is that they are based on readily accessible data that are available for all areas. However, it should be noted that there are some groups for which the census does not provide reliable information - in particular the homeless.

Until 1991, the ten-yearly census was completed in relation to households and so, by definition, omitted the homeless. While there is likely to be a relationship between homelessness and the change of address factor, it is both uncertain and indirect. However, for the first time, the 1991 census attempted to enumerate certain sub-groups of homeless individuals. Street workers who had had regular contact with the 'literal' (i.e. roofless) homeless were enrolled as enumerators to facilitate this process. It should be remembered that the roofless, while highly visible and politically embarrassing, comprise only a fraction of the total number of homeless. Further, it has been argued that this simple headcounting approach is not the best suited to estimate the size of the homeless population (Susser et al, 1989).

Awareness of the size of the homeless population is important for those planning mental health services, as the homeless are known both to suffer an excess of mental disorder (Tessler \& Dennis, 1989) and to be heavy users of mental health services (Fisher $e t$ al, 1990). As a result, in accordance with 'Working for Patients' (Department of Health, 1989), it is incumbent on health authorities to make specific attempts to estimate the size and mental health needs of their local homeless population.

This information must then be utilised in a creative fashion to ensure that the health needs of the homeless are met. For example, there is no evidence that the homeless are unable to make use of existing services. Any excess unmet need is likely to reflect patterns of use of service that are so chaotic as to be 